\title{
On a fractional-order delay Mackey-Glass equation
}

\author{
Ahmed MA El-Sayed ${ }^{1 *}$, Sanaa M Salman² and Naemaa A Elabd ${ }^{3}$
}

${ }^{\text {*Correspondence: }}$ amasayed@gmail.com

'Department of Mathematics, Faculty of Science, Alexandria University, Alexandria, Egypt Full list of author information is available at the end of the article

\begin{abstract}
In this paper, a fractional-order Mackey-Glass equation with constant delay is considered. The local stability of the fixed points is analyzed. Moreover, a discretization process is applied to convert the fractional-order delay equation to its discrete analog. A numerical simulation including Lyapunov exponent, phase diagrams, bifurcation, and chaos is carried out using Matlab to ensure theoretical results and to reveal more complex dynamics of the equation after discretization.
\end{abstract}

Keywords: fractional-order delay Mackey-Glass equations; fixed points; local stability; discretization; Lyapunov exponent; bifurcation; chaos

\section{Introduction}

Delay differential equations (DDEs) arise in the mathematical description of systems whose time evolution depends explicitly on a past state of the system, as for example in the case of delayed feedback. Neural systems [1], respiration regulation [2], agricultural commodity markets [3], nonlinear optics, and neutrophil populations in the blood [2] are but a few systems in which delayed feedback leads naturally to a description in terms of a delay differential equation. We will restrict our attention to systems modeled by evolutionary delay equations that can be expressed in the form

$$
x^{\prime}(t)=f(x(t), x(t-\tau)), \quad x(t) \in \Re^{n}, t \geq 0 .
$$

Here the 'state' of the system at time $t$ is $x(t)$, whose rate of change depends explicitly, via the function $f$, on the past state $x(t-\tau)$ where $\tau$ is a fixed time delay. More general delay equations might be considered: multiple time delays, variable time delays, continuously distributed delays, and higher derivatives all arise in applications and lead to more complicated evolution equations. Nevertheless, equations of the form (1.1) constitute a sufficiently broad class of systems to be of practical importance, and they will provide adequate fodder for the types of problems we wish to consider.

DDEs arise in many areas of mathematical modeling: for example, population dynamics (taking into account the gestation times), infectious diseases (accounting for the incubation periods), physiological and pharmaceutical kinetics (modeling, for example, the body's reaction to $\mathrm{CO}_{2}$, etc. in circulating blood), chemical kinetics (such as mixing reactants), the navigational control of ships and aircraft (with, respectively, large and short lags), and more general control problems (see for example [4-6]).

(c) 2016 El-Sayed et al. This article is distributed under the terms of the Creative Commons Attribution 4.0 International License (http://creativecommons.org/licenses/by/4.0/), which permits unrestricted use, distribution, and reproduction in any medium, provided you give appropriate credit to the original author(s) and the source, provide a link to the Creative Commons license, and indicate if changes were made. 
On the other hand, fractional calculus is a generalization of classical differentiation and integration to arbitrary (non-integer) order [7-9]. Many mathematicians and applied researchers have tried to model real processes using fractional calculus [10-16]. In recent years differential equations with fractional-order have attracted many researchers because of their applications in many areas of science and engineering. Analytical and numerical techniques have been implemented to study such equations. The fractional calculus has allowed the operations of integration and differentiation to be applied for any fractional order [17-21].

We recall the basic definitions (Caputo) and properties of fractional-order differentiation and integration.

Definition 1 The fractional integral of order $\beta \in \mathbb{R}^{+}$of the function $f(t), t>0$ is defined by

$$
I_{a}^{\beta} f(t)=\int_{0}^{t} \frac{(t-s)^{\beta-1}}{\Gamma(\beta)} f(s) d s,
$$

and the fractional derivative of order $\alpha \in(n-1, n)$ of $f(t), t>0$ is defined by

$$
D_{a}^{\alpha} f(t)=I^{n-\alpha} D^{n} f(t), \quad D=\frac{d}{d t} .
$$

In addition, the following results are the main features in fractional calculus. Let $\beta, \gamma \in$ $\mathbb{R}^{+}, \alpha \in(0,1)$ :

- $I_{a}^{\beta}: L^{1} \rightarrow L^{1}$, and if $f(x) \in L^{1}$, then $I_{a}^{\gamma} I_{a}^{\beta} f(x)=I_{a}^{\gamma+\beta} f(x)$.

- $\lim _{\beta \rightarrow n} I_{a}^{\beta} f(x)=I_{a}^{n} f(x)$ uniformly on $[a, b], n=1,2,3, \ldots$, where $I_{a}^{1} f(x)=\int_{a}^{x} f(s) d s$.

- $\lim _{\beta \rightarrow 0} I_{a}^{\beta} f(x)=f(x)$ weakly.

- If $f(x)$ is absolutely continuous on $[a, b]$, then $\lim _{\alpha \rightarrow 1} D_{a}^{\alpha} f(x)=\frac{d f(x)}{d x}$.

The Mackey-Glass equation is a nonlinear time delay differential equation, which was proposed as a model of hematopoiesis, given by

$$
\frac{d x}{d t}=\frac{\rho x_{\tau}}{1+x_{\tau}^{c}}-\gamma x
$$

where $\gamma, c, \rho, \tau$ are real parameters, and $x_{\tau}$ represents the value of the variable $x$ at time ( $t-$ $\tau)$. Depending on the values of the parameters, this equation displays a range of periodic and chaotic dynamics.

In this work, we will show that considering a fractional-order derivative with delay in equation (1.2) will exhibit more complex and richer dynamics.

Consider the fractional-order delay Mackey-Glass equation given in the form

$$
D^{\alpha} x(t)=\frac{\rho x(t-\tau)}{1+x(t-\tau)^{c}}-x(t-\tau), \quad t \in(0, T]
$$

with the initial condition

$$
x(0)=x_{0},
$$

where $\alpha \in(0,1], \rho \in R^{+}$, and $c>0$. In equation (1.3), we consider delay in the last term. 


\section{Discretization process}

In this part, we apply the discretization process represented in [22, 23], and [24] for discretizing the delay fractional-order Mackey Glass equation with piecewise constant arguments given by

$$
D^{\alpha} x(t)=\frac{\rho x\left(\left[\frac{t}{r}\right] r-\tau\right)}{1+x^{c}\left(\left[\frac{t}{r}\right] r-\tau\right)}-x\left(\left[\frac{t}{r}\right] r-\tau\right)
$$

with initial condition (1.4).

The steps of the discretization process are as follows.

Let $t \in[0, r)$, then $\frac{t}{r} \in[0,1)$. That is,

$$
D^{\alpha} x(t)=\frac{\rho x_{0}(-\tau)}{1+x_{0}^{c}(-\tau)}-x_{0}(-\tau)
$$

and the solution of (2.2) is given by

$$
\begin{aligned}
x(t) & =x_{0}+I^{\alpha}\left(\frac{\rho x_{0}(-\tau)}{1+x_{0}^{c}(-\tau)}-x_{0}(-\tau)\right) \\
& =x_{0}+\left(\frac{\rho x_{0}(-\tau)}{1+x_{0}^{c}(-\tau)}-x_{0}(-\tau)\right) \int_{0}^{t} \frac{(t-s)^{\alpha-1}}{\Gamma(\alpha)} d s \\
& =x_{0}+\frac{t^{\alpha}}{\Gamma(1+\alpha)}\left(\frac{\rho x_{0}(-\tau)}{1+x_{0}^{c}(-\tau)}-x_{0}(-\tau)\right) .
\end{aligned}
$$

Let $t \in[r, 2 r)$, then $\frac{t}{r} \in[1,2)$. That is,

$$
D^{\alpha} x(t)=\frac{\rho x_{1}(r-\tau)}{1+x_{1}^{c}(r-\tau)}-x_{1}(r-\tau), \quad t \in[r, 2 r)
$$

and the solution of (2.3) is given by

$$
\begin{aligned}
x_{2}(t) & =x_{1}(r)+I_{r}^{\alpha}\left(\frac{\rho x_{1}(r-\tau)}{1+x_{1}^{c}(r-\tau)}-x_{1}(r-\tau)\right) \\
& =x_{1}(r)+\left(\frac{\rho x_{1}(r-\tau)}{1+x_{1}^{c}(r-\tau)}-x_{1}(r-\tau)\right) \int_{r}^{t} \frac{(t-s)^{\alpha-1}}{\Gamma(\alpha)} d s \\
& =x_{1}(r)+\frac{(t-r)^{\alpha}}{\Gamma(1+\alpha)}\left(\frac{\rho x_{1}(r-\tau)}{1+x_{1}^{c}(r-\tau)}-x_{1}(r-\tau)\right) .
\end{aligned}
$$

Repeating the process we can easily get

$$
x(t)=x_{n}(n r)+\frac{(t-n r)^{\alpha}}{\Gamma(1+\alpha)}\left(\frac{\rho x_{n}(n r-\tau)}{1+x_{n}^{c}(n r-\tau)}-x_{n}(n r-\tau)\right), \quad t \in[n r,(n+1) r) .
$$

Let $t \rightarrow(n+1) r$, we obtain the discretization

$$
x_{n+1}(n+1)=x_{n}+\frac{(r)^{\alpha}}{\Gamma(1+\alpha)}\left(\frac{\rho x_{n}(r-\tau)}{1+x_{n}^{c}(r-\tau)}-x_{n}(r-\tau)\right) .
$$

It is worth to pay attention here that Euler's discretization method is an approximation for the derivative while the predictor-corrector method is an approximation for the integral. 
However, our proposed discretization method here is an approximation for the right-hand side of the system under consideration as is pretty clear from (2.4). Moreover, we have noticed that when $\alpha \rightarrow 1$, the discretization will be Euler's discretization.

In the following, we will discuss two cases of the delay: Case I: $\tau=r$, and Case II: $\tau=2 r$.

\section{Case I: $\tau=r$}

In this case we have a second-order difference equation given by

$$
x_{n+1}=x_{n}+\frac{r^{\alpha}}{\Gamma(1+\alpha)}\left(\frac{\rho x_{n-1}}{1+x_{n-1}^{c}}-x_{n-1}\right) .
$$

\section{Existence and stability of fixed points}

To find the fixed points of system (3.1), we first split it into two first-order difference equations as follows:

$$
\begin{aligned}
& x_{n+1}=x_{n}+\frac{r^{\alpha}}{\Gamma(1+\alpha)}\left(\frac{\rho y_{n}}{1+y_{n}^{c}}-y_{n}\right), \\
& y_{n+1}=x_{n} .
\end{aligned}
$$

- For all values of the parameter $\rho$, system (3.2) has one fixed point, namely, fix $x_{1}=(0,0)$.

- For $\rho>1$, we have an additional fixed point, which is $\mathrm{fix}_{2}=(\sqrt[c]{\rho-1}, \sqrt[c]{\rho-1})$.

In order to study the local stability of these fixed points, we need the moduli of the eigenvalues of the Jacobian matrix evaluated at each of the fixed points [25]. The Jacobian matrix of system (3.2) evaluated at any fixed point $(x, y)$ is obtained by

$$
J=\left(\begin{array}{cc}
1 & \frac{r^{\alpha}}{\Gamma(1+\alpha)}\left(\frac{\rho+\rho y^{c}(1-c)}{\left(1+y^{c}\right)^{2}}-1\right) \\
1 & 0
\end{array}\right) .
$$

The eigenvalues associated to the Jacobian matrix are

$$
\lambda_{1,2}=0.5 \pm 0.5 \sqrt{1+4 R(s-1)}
$$

where

$$
R=\frac{r^{\alpha}}{\Gamma(1+\alpha)}, \quad S=\frac{\rho+\rho y^{c}(1-c)}{\left(1+y^{c}\right)^{2}} .
$$

The fixed points fix $x_{1}, f_{2}$ of the system equation (3.1) are stable if $\left|\lambda_{i}\right|<1, i=1,2$. In order to study the qualitative behavior of the solution of system (3.2) we rely on the Jury criteria given generally by

1. $F:=1+T+D>0$,

2. $T C:=1-T+D>0$,

3. $H:=1-D>0$,

where the trace and determinant of the Jacobian matrix are given, respectively, by

$$
\begin{aligned}
& T: \operatorname{Tr}(J)=J_{11}+J_{22}, \\
& D: \operatorname{Det}(J)=J_{11} J_{22}-J_{12} J_{21} .
\end{aligned}
$$


Proposition 1 The fixed point $\mathrm{fix}_{1}$ is locally asymptotically stable if $\rho<(1+2 / R)$, and losses stability via a flip bifurcation when $\rho>1$ and via a Neimark-Sacker bifurcation when $\rho>\frac{r^{\alpha}-\Gamma(1+\alpha)}{r^{\alpha}}$.

Proof The Jacobian matrix at the first fixed point fix $x_{1}$ is obtained by

$$
J=\left(\begin{array}{cc}
1 & \frac{r^{\alpha}}{\Gamma(1+\alpha)}(\rho-1) \\
1 & 0
\end{array}\right),
$$

which has two eigenvalues,

$$
\lambda_{1,2}=0.5 \pm 0.5 \sqrt{1+4 R(\rho-1)} .
$$

According to the Jury criteria [26,27], where $T=1, D=\frac{r^{\alpha}(1-\rho)}{\Gamma(1+\alpha)}$, the first condition is always satisfied, while the second and third may be violated. That is, the fixed point fix 1 loses stability via a flip bifurcation when $\rho>1$, and via a Neimark-Sacker bifurcation when $\rho>$ $\frac{r^{\alpha}-\Gamma(1+\alpha)}{r^{\alpha}}$.

Proposition 2 The fixed point fix $x_{2}$ of system (3.2) is stable if $\rho<\frac{c R}{2+c R}$, and it loses stability via a pitchfork bifurcation if $\rho>\frac{c R}{2+c R}$, via a flip bifurcation if $\rho>1$, and via a NeimarkSacker bifurcation if $\rho<\frac{c R}{c R-1}$.

Proof Calculating the Jacobian matrix at the second fixed point fix $x_{2}$ of system (3.2) we obtain

$$
J=\left(\begin{array}{cc}
1 & \frac{r^{\alpha} c(1-\rho)}{\rho \Gamma(1+\alpha)} \\
1 & 0
\end{array}\right),
$$

which has two eigenvalues,

$$
\lambda_{1,2}=0.5 \pm 0.5 \sqrt{1+\frac{4 R c(1-\rho)}{\rho}},
$$

where the trace and determinant of $J\left(\mathrm{fix}_{2}\right)$ are given, respectively, by

$$
T=1, \quad D=-R c\left(\frac{1-\rho}{\rho}\right) .
$$

According to the Jury criteria, the three conditions may be all violated. That is, fix $x_{2}$ loses stability via a pitchfork bifurcation if $\rho>\frac{c R}{2+c R}$, via a flip bifurcation if $\rho>1$, and via a Neimark-Sacker bifurcation if $\rho<\frac{c R}{c R-1}$.

4 Case II: $\tau=2 r$

In this section, we take the delay to be $\tau=2 r$ in equation (1.3). Applying the discretization process we end up with a system of third-order difference equations given by

$$
x_{n+1}=x_{n}+\frac{r^{\alpha}}{\Gamma(1+\alpha)}\left(\frac{\rho x_{n-2}}{1+x_{n-2}^{c}}-x_{n-2}\right) \text {. }
$$


To study the fixed points of system (4.1) we first split it into three first-order difference equations as follows:

$$
\begin{aligned}
& x_{n+1}=x_{n}+\frac{r^{\alpha}}{\Gamma(1+\alpha)}\left(\frac{\rho y_{n}}{1+z_{n}^{c}}-z_{n}\right), \\
& y_{n+1}=x_{n}, \\
& z_{n+1}=y_{n} .
\end{aligned}
$$

In the following, we study the local stability of the fixed points of the system (4.2).

\section{Existence and stability of fixed points}

System (4.2) has the following fixed points:

- For all parameter values, there is only one fixed point fix $x_{1}=(0,0,0)$.

- For $\rho>1$, there is an additional fixed point fix $x_{2}=(\sqrt[c]{\rho-1}, \sqrt[c]{\rho-1}, \sqrt[c]{\rho-1})$.

By considering a Jacobian matrix for one of these fixed points and calculating their eigenvalues, we can investigate the stability of each fixed point based on the roots of the system characteristic equation. The Jacobian matrix is given by

$$
J=\left(\begin{array}{ccc}
1 & 0 & R(S-1) \\
1 & 0 & 0 \\
0 & 1 & 0
\end{array}\right),
$$

where $R=\frac{r^{\alpha}}{\Gamma(1+\alpha)}, S=\frac{\rho+\rho z^{c}(1-c)}{\left(1+z^{c}\right)^{2}}$.

Linearizing the system (4.2) about fix $x_{1}$ yields the following characteristic equation:

$$
P(\lambda)=\lambda^{3}-\lambda^{2}-R(\rho-1)
$$

Let

$$
a_{0}=1, \quad a_{1}=-1, \quad a_{2}=0, \quad a_{3}=-R(\rho-1) .
$$

From the Jury test, if $P(1)>0, P(-1)<0$, and $a_{3}<1,\left|b_{3}\right|>b_{1}, c_{3}>\left|c_{2}\right|$, where $b_{3}=1-a_{3}^{2}$, $b_{2}=a_{1}-a_{3} a_{2}, b_{1}=a_{2}-a_{3} a_{1}, c_{3}=b_{3}^{2}-b_{1}^{2}$, and $c_{2}=b_{3} b_{2}-b_{1} b_{2}$, then the roots of $P(\lambda)$ satisfy $\lambda<1$ and thus fix 1 is asymptotically stable.

The first condition gives $\rho<1$, while the second condition gives

$$
\rho>\frac{r^{\alpha}-2 \Gamma(\alpha+1)}{r^{\alpha}}
$$

The remaining conditions give the following inequalities:

- $a_{3}<1 \Rightarrow \rho>1-\frac{1}{R}$

- $\left|b_{3}\right|>b_{1} \Rightarrow\left(1-(R-\rho R)^{2}\right)>(R-\rho R)$,

- $c_{3}>\left|c_{2}\right| \Rightarrow\left(1-(R-\rho R)^{2}\right)^{2}-(R-\rho R)^{2}>(R-\rho R)^{2}-1+(R-\rho R)$.

Linearizing the system (4.2) about fix $x_{2}$ yields the following characteristic equation:

$$
P(\lambda)=\lambda^{3}-\lambda^{2}-\frac{c R(1-\rho)}{\rho} .
$$


Let

$$
a_{0}=1, \quad a_{1}=-1, \quad a_{2}=0, \quad a_{3}=-\frac{c R(1-\rho)}{\rho} .
$$

From the Jury test, if $P(1)>0, P(-1)<0$, and $a_{3}<1,\left|b_{3}\right|>b_{1}, c_{3}>\left|c_{2}\right|$, where $b_{3}=1-a_{3}^{2}$, $b_{2}=a_{1}-a_{3} a_{2}, b_{1}=a_{2}-a_{3} a_{1}, c_{3}=b_{3}^{2}-b_{1}^{2}$, and $c_{2}=b_{3} b_{2}-b_{1} b_{2}$, then the roots of $P(\lambda)$ satisfy $\lambda<1$ and thus $\mathrm{fix}_{1}$ is asymptotically stable.

We are going to check these conditions at fix $x_{2}$ :

- $p(1)>0 \Rightarrow \rho>1$,

- $p(-1)<0 \Rightarrow \rho<\frac{c r^{\alpha}}{c r^{\alpha}-2 \Gamma(\alpha+1)}$,

- $a_{3}<1 \Rightarrow \rho<\frac{c r^{\alpha}}{c r^{\alpha}-\Gamma(\alpha+1)}$

- $\left|b_{3}\right|>b_{1} \Rightarrow\left(1+\frac{c^{2} R^{2}}{\rho^{2}}(1-\rho)^{2}\right)>\frac{c R(\rho-1)}{\rho}$.

Thus, any condition may be violated resulting in instability of fix $x_{2}$.

\section{Numerical simulation}

In this section, a numerical simulation is carried out with the aid of Matlab to illustrate our theoretical results and to reveal the more complex dynamics of equation (1.2) in the two cases $\tau=r$ and $\tau=2 r$. In all numerical simulations, we take $c=6$, and $r=0.5$. First of all, let us consider system (3.2). Indeed, if one is interested in determining whether a dynamical system is chaotic or not, often just a few of the largest Lyapunov characteristic exponents (LCEs) may provide the answer. This actually is so because a positive LCE is a good indicator for chaos. Since for non-chaotic systems all LCEs are non-positive, the presence of a positive LCE has often been used to help determine if a system is chaotic or not. In this paper, we compute the LCEs via the Householder QR-based methods described in [28]. For system (3.2), we get when $\alpha=0.95, c=6$, and $r=0.5$, LCE1 $=0.3397$, and LCE2 $=0.185$ as shown in Figure 1 . We vary the parameter $\rho$ and fix the other parameters, $c, r$, and $\alpha$. Bifurcation diagrams of system (3.2) are also shown in Figure 1 for different values of the fractional-order parameter $\alpha$. If we consider $\alpha=0.95$, it is shown that the fixed point fix $\mathrm{x}_{1}(0,0)$ is stable if $\rho<1$, and at $\rho>1$ it losses stability via a flip bifurcation. Afterwards, a stable periodic solution of period 2 appears, then the periodic solution of period 4 becomes unstable, and a periodic solution of period 8 appears and chaos happens. Figure 2 shows the different phase plane for system (3.2) for $\alpha=0.95$. For $\rho=1.55$, Figure 2(a) shows an invariant closed curve bifurcating from fix $1(0,0)$, while for $\rho=3.6$, Figure 2(b) shows a chaotic attractor. Now we vary the parameter $\alpha$ from 0 to 1 and fix $\rho$ to plot the bifurcation diagram for system (3.2) as a function of $\alpha$ as shown in Figure 3.

Next, we turn to the second case, when $\tau=2 r$. Figure 4 shows the bifurcation diagram for system (4.2) as a function of $\rho$. If $\rho=0.95$, the figure shows that the fixed point fix $x_{1}$ becomes unstable when $\rho>1$ as it loses stability via a flip bifurcation. Then the stable period-2 orbit appears at $\rho=1.2$, which in turn loses stability; then chaos appears. Finally, Figure 5 shows the phase plane for system (4.2) for $\alpha=0.95$. Figure 5(a) shows a stable fixed point fix $x_{1}$ for $\rho=0.6$, Figure 5(b) shows a double scroll for $\rho=1.2$, and Figure 5(c), (d) shows chaotic attractors for $\rho=2$, and $\rho=2.7$, respectively.

\section{Conclusion}

In this paper, the dynamic behavior of a fractional-order delay Mackey-Glass equation is investigated after applying a discretization process to it. We have considered two different 


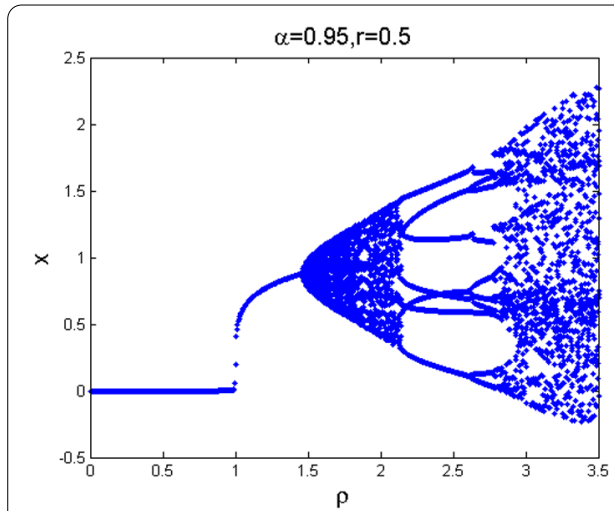

(a)

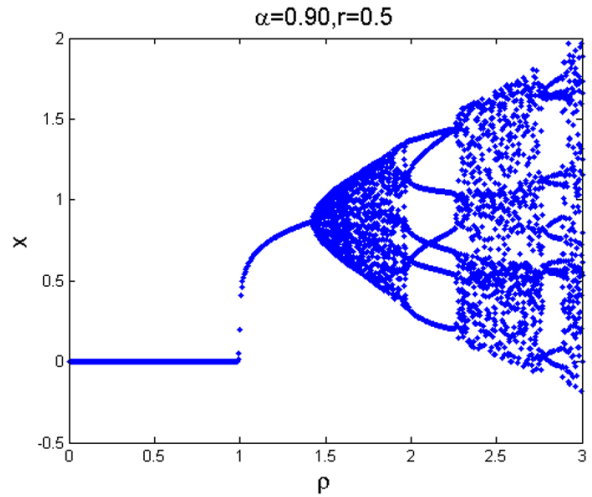

(c)

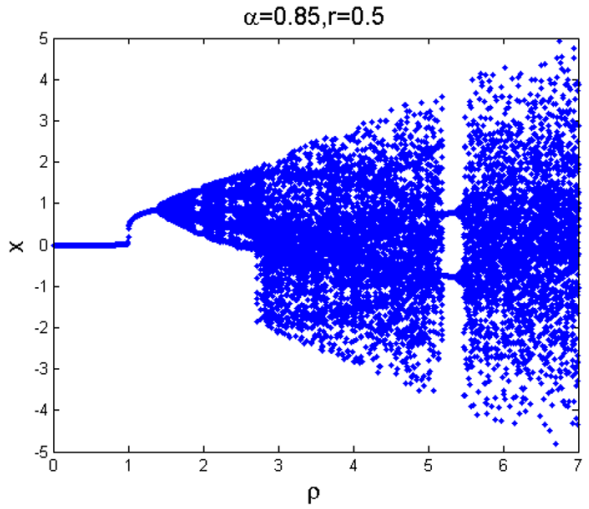

(e)

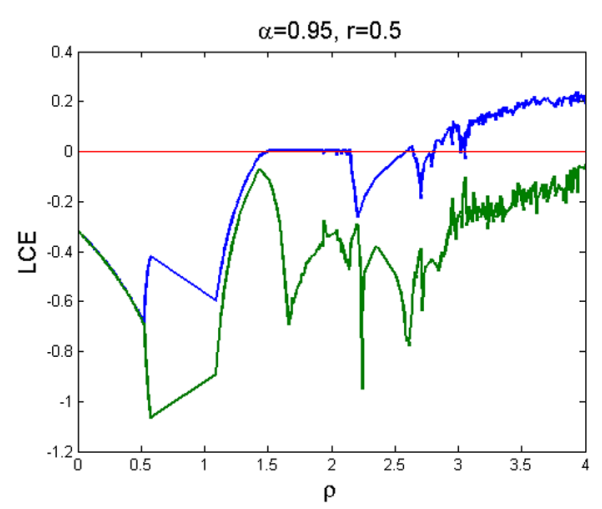

(b)

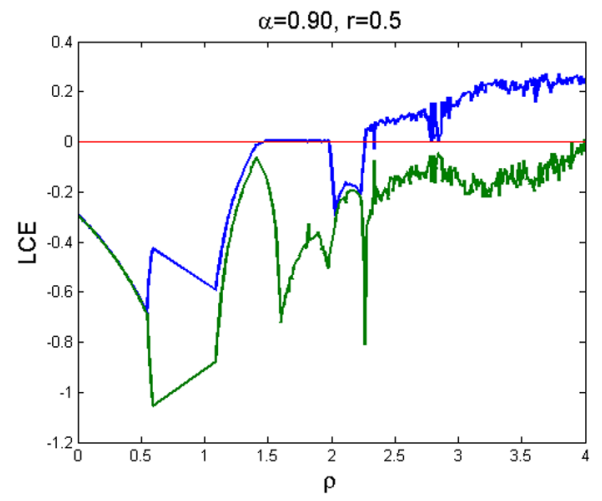

(d)

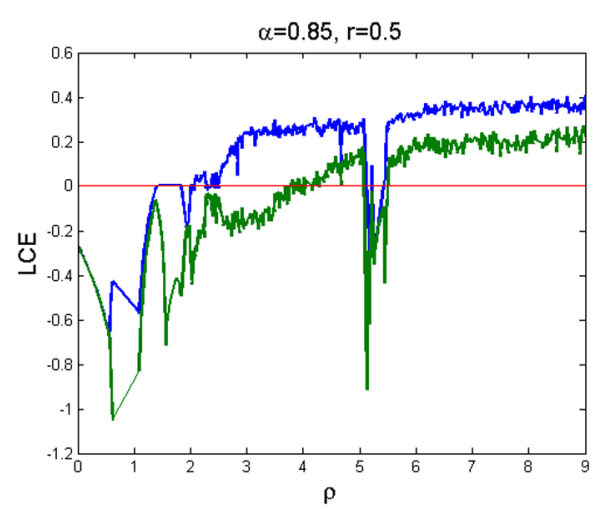

(f)

Figure 1 Bifurcation diagram and Lyapunov exponent for system (3.2) with different values of the fractional-order parameter $\alpha, c=6$ and $r=0.5$.

cases for the delay $\tau$, the first is when $\tau=r$, and the second is when $\tau=2 r$, where $r$ is the discretization parameter. Stability of the fixed points and local bifurcations of fixed points of the discretized systems in the two cases was are analyzed. A numerical simulation was carried out to ensure our theoretical analysis and to reveal the more complex dynamics of the system. 


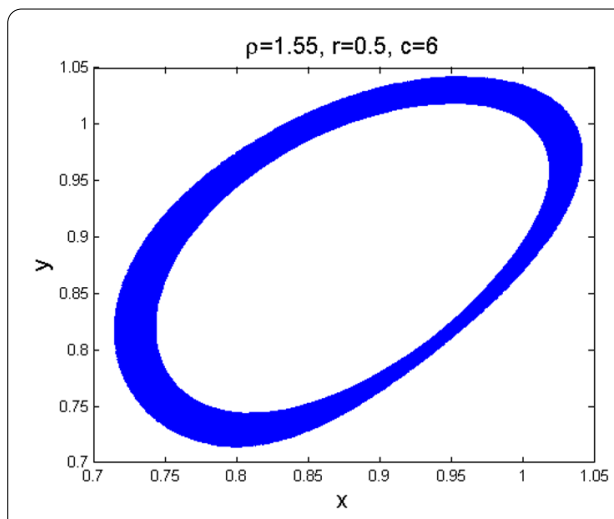

(a)

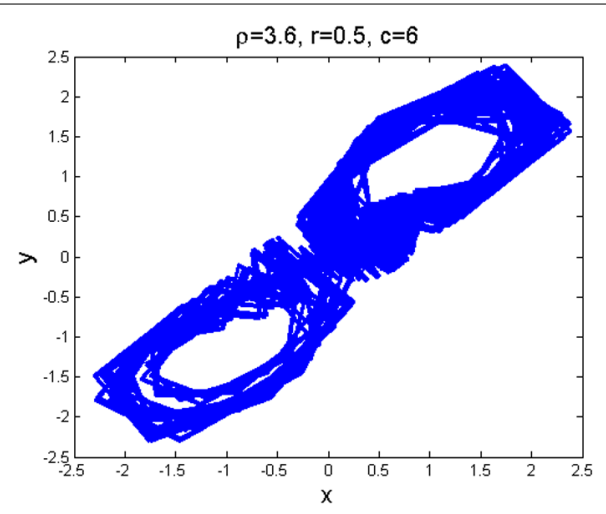

(b)

Figure 2 Phase plane for system (3.2) with $\alpha=0.95, c=6$ and $r=0.5$.

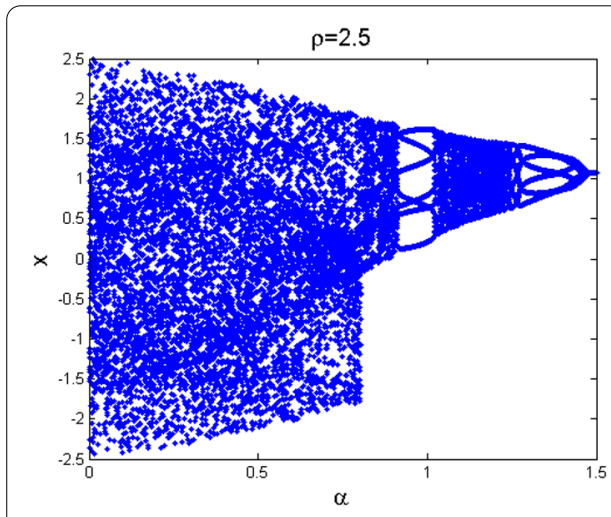

(a)

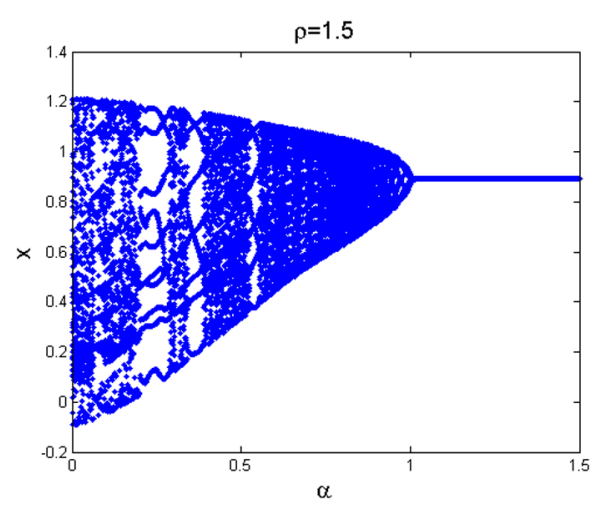

(b)

Figure 3 Bifurcation diagrams for system (3.2) as a function of $\alpha$.

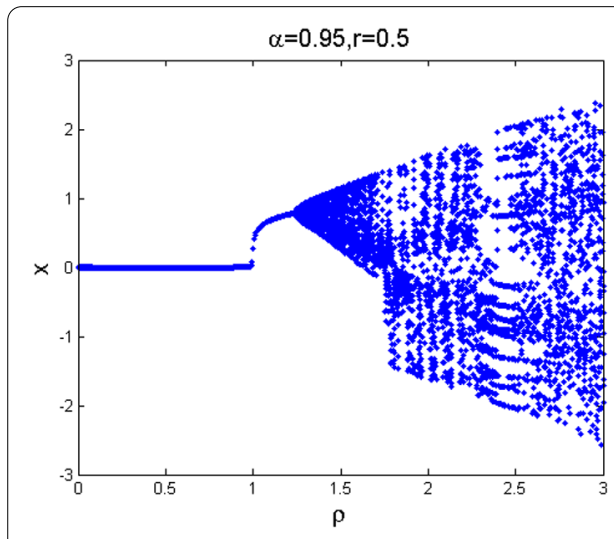

(a)

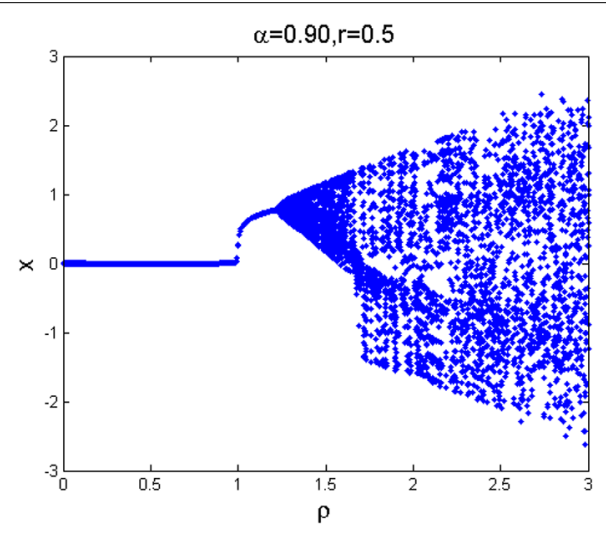

(b)

Figure 4 Bifurcation diagram and chaos for system (4.2) as a function of $\rho$. 


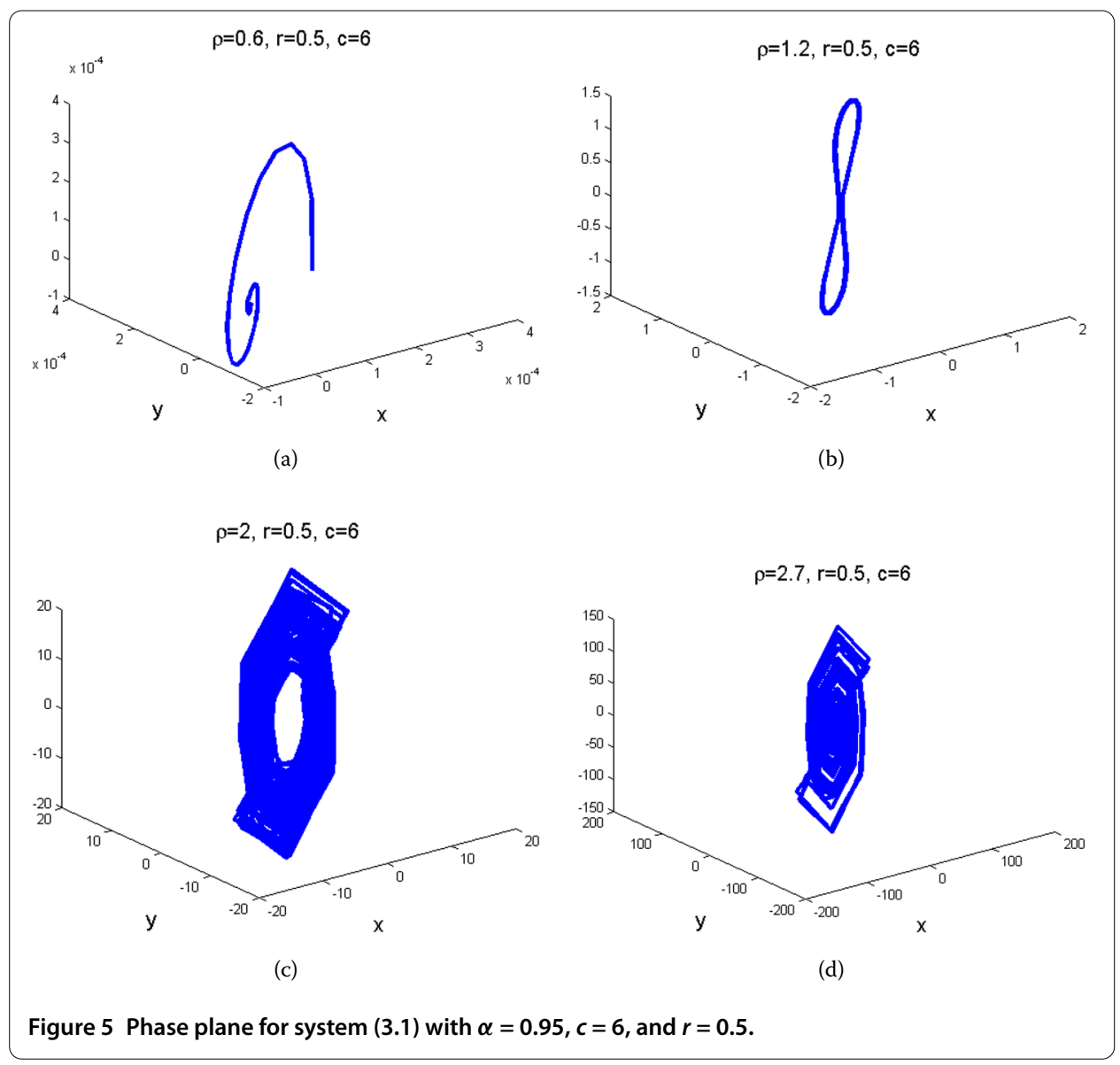

\section{Competing interests}

The authors declare that they have no competing interests.

\section{Authors' contributions}

The authors declare that the study was realized in collaboration with the same responsibility. All authors read and approved the final manuscript.

\section{Author details}

'Department of Mathematics, Faculty of Science, Alexandria University, Alexandria, Egypt. ${ }^{2}$ Faculty of Education, Alexandria University, Alexandria, Egypt. ${ }^{3}$ Faculty of Science, Alexandria University, Alexandria, Egypt.

\section{Acknowledgements}

The authors would like to thank the referees of this manuscript for their valuable comments and suggestions.

Received: 10 February 2016 Accepted: 11 May 2016 Published online: 18 May 2016

\section{References}

1. an der Heiden, U: Delays in physiological systems. J. Math. Biol. 8, 345-364 (1979)

2. Glass, L, Mackey, MC: Pathological conditions resulting from instabilities in physiological control systems. Ann. N.Y. Acad. Sci. 316, 214-235 (1979)

3. Mackey, MC: Commodity price fluctuations: price dependent delays and non- linearities as explanatory factors. J. Econ. Theory 48(2), 497-509 (1989)

4. Baleanu, D, Magin, RL, Bhalekar, S, Daftardar-Gejji, V: Chaos in the fractional order nonlinear Bloch equation with delay. Commun. Nonlinear Sci. Numer. Simul. 25(1-3), 41-49 (2015)

5. Wu, G-C, Baleanu, D: Discrete chaos in fractional delayed logistic maps. Nonlinear Dyn. 80(4), 1697-1703 (2015)

6. Jarad, F, Abdeljawad, T, Baleanu, D: Higher order fractional variational optimal control problems with delayed arguments. Appl. Math. Comput. 218(18), 9234-9240 (2012)

7. Kilbas, AA, Srivastava, HM, Trujillo, JJ: Theory and Applications of Fractional Differential Equations. Elsevier, Amsterdam (2006)

8. Podlubny, I: Fractional Differential Equations. Mathematics in Science and Engineering, vol. 198. Academic Press, San Diego (1999) 
9. Miller, KS, Ross, B: An Introduction to the Fractional Calculus and Fractional Differential Equation. Wiley, New York (1993)

10. Yang, XJ, Srivastava, HM, Cattani, C: Local fractional homotopy perturbation method for solving fractal partial differential equations arising in mathematical physics. Rom. Rep. Phys. 67(3), 752-761 (2015)

11. Yang, XJ, Srivastava, HM: An asymptotic perturbation solution for a linear oscillator of free damped vibrations in fractal medium described by local fractional derivatives. Commun. Nonlinear Sci. Numer. Simul. 29(1), 499-504 (2015)

12. Yang, XJ, Baleanu, D, Srivastava, HM: Local fractional similarity solution for the diffusion equation defined on Cantor sets. Appl. Math. Lett. 47, 54-60 (2015)

13. Wang, J, Ye, Y, Pan, X, Gao, X, Zhuang, C: Fractional zero-phase filtering based on the Riemann-Liouville integral. Signal Process. 98(5), 150-157 (2014)

14. Wang, J, Ye, Y, Gao, Y, Gao, X, Qian, S: Fractional compound integral with application to ECG signal denoising. Circuits Syst. Signal Process. 34, 1915-1930 (2015)

15. Wang, J, Ye, Y, Pan, X, Gao, X: Parallel-type fractional zero-phase filtering for ECG signal denoising. Biomed. Signal Process. Control 18, 36-41 (2015)

16. Wang, J, Ye, Y, Gao, X: Fractional $90^{\circ}$ phase-shift filtering based on the double-sided Grunwald-Letnikov differintegrator. IET Signal Process. 9(4), 328-334 (2015)

17. Bhrawy, AH, Alhamed, YA, Baleanu, D, Al-Zahrani, AA: New spectral techniques for systems of fractional differential equations using fractional-order generalized Laguerre orthogonal functions. Fract. Calc. Appl. Anal. 17, 1137-1157 (2014)

18. Hafez, RM, Ezz-Eldien, SS, Bhrawy, AH, Ahmed, EA, Baleanu, D: A Jacobi Gauss-Lobatto and Gauss-Radau collocation algorithm for solving fractional Fokker-Planck equations. Nonlinear Dyn. 82(3), 1431-1440 (2015)

19. Bhrawy, AH, Hafez, RM, Alzahrani, E, Baleanu, D, Alzahrani, AA: Generalized Laguerre-Gauss-Radau scheme for the first order hyperbolic equations in a semi-infinite domain. Rom. J. Phys. 60, 918-934 (2015)

20. Bhrawy, AH, Abdelkawy, MA, Alzahrani, AA, Baleanu, D, Alzahrani, E: A Chebyshev-Laguerre Gauss-Radau collocation scheme for solving time fractional sub-diffusion equation on a semi-infinite domain. Proc. Rom. Acad., Ser. A 16, 490-498 (2015)

21. Doha, EH, Bhrawy, AH, Baleanu, D, Ezz-Eldien, SS, Hafez, RM: An efficient numerical scheme based on the shifted orthonormal Jacobi polynomials for solving fractional optimal control problems. Adv. Differ. Equ. 2015, 15 (2015)

22. El Raheem, ZF, Salman, SM: On a discretization process of fractional-order logistic differential equation. J. Egypt. Math. Soc. 22, 407-412 (2014)

23. El-Sayed, AMA, El-Raheem, ZF, Salman, SM: Discretization of forced Duffing system with fractional-order damping. Adv. Differ. Equ. 2014, 66 (2014)

24. El-Sayed, AMA, Salman, SM: On a discretization process of fractional-order Riccati differential equation. J. Fract. Calc. Appl. 4(2), 251-259 (2013)

25. Elaidy, SN: An Introduction to Difference Equations, 3rd edn. Undergraduate Texts in Mathematics. Springer, New York (2005)

26. Palis, J, Takens, F: Hyperbolicity and Sensitive Chaotic Dynamics and Homoclinic Bifurcation. Cambridge University Press, Cambridge (1993)

27. Puu, T: Attractors, Bifurcation and Chaos: Nonlinear Phenomena in Economics. Springer, Berlin (2000)

28. Udwadia, FE, von Bremen, $\mathrm{H}$ : A note on the computation of the largest $p$-Lyapunov characteristic exponents for nonlinear dynamical systems. J. Appl. Math. Comput. 114, 205-214 (2000)

\section{Submit your manuscript to a SpringerOpen ${ }^{\circ}$ journal and benefit from:}

- Convenient online submission

Rigorous peer review

- Immediate publication on acceptance

- Open access: articles freely available online

- High visibility within the field

- Retaining the copyright to your article 